\title{
A-CONTRARIO LOCALIZATION OF EPILEPTOGENIC ZONES IN SPECT IMAGES
}

\author{
Cecilia Aguerrebere, Pablo Sprechmann and Pablo Musé
}

\author{
Universidad de la República \\ Instituto de Ingeniera Eléctrica \\ Montevideo, Uruguay, 11300
}

\author{
Rodolfo Ferrando
}

\author{
Universidad de la República \\ Centro de Medicina Nuclear \\ Montevideo, Uruguay, 11800
}

\begin{abstract}
In refractory epilepsy, the goal of neuroimaging is to localize the region of seizure onset. Tracers that accumulate and remain fixed proportional to regional cerebral blood flow $(\mathrm{rCBF})$ at the time of injection are used to obtain SPECT images of the brain activity during and between seizures. The most used technique for detecting the epileptogenic zone (EZ) is to threshold the co-registered and normalized subtraction of these two images. This method has proven to be very useful but has some disadvantages: result depends on the selected threshold and abundance of false detections. In this paper we propose an a-contrario algorithm for detecting regions of the brain with significant changes in the rCBF using two SPECT images. This new method arises from formal deduction and no arbitrary parameters are involved. Comparisons of both methodologies on six patients are presented. The proposed algorithm shows good results in all cases and is more robust than the thresholding method.
\end{abstract}

Index Terms - A-contrario detection, SPECT, refractory epilepsy

\section{INTRODUCTION}

Epilepsy is a common neurological disorder. A large number of patients have seizures that are refractory to therapy. Medically refractory seizure disorders in children may have poor neuro-developmental outcomes. Surgery is the only option in these situations and requires a previous precise localization of the EZ.

Functional neuroimaging techniques, such as PET or SPECT, are useful for this purpose [1]. A radioactive tracer is given to the patient by intravenous injection. Brain perfusion SPECT tracers are lipophilic substances with free diffusion across the blood brain barrier that have a long retention time in the brain. Two ${ }^{99 m}$ technetium( ${ }^{99 m} \mathrm{Tc}$ )-labeled agents are most commonly used: ${ }^{99 m}$ Tc-HMPAO (hexamethylpropylene amine oxime) and ${ }^{99 m}$ Tc-ECD (ethylcysteinate dimer). Tracer distribution is proportional to $\mathrm{rCBF}$ at the moment of administration and stays stable up to 4 to 6 hours. Gamma rays emitted by the tracer are detected with a gamma camera about an hour after injection. This produces a 3D reconstruction of $\mathrm{rCBF}$ distribution at the moment the patient was injected. An ictal SPECT can be obtained when the tracer is injected during a seizure, while the patient must be injected in-between seizures for an inter-ictal SPECT. EZ appear in the ictal scan as spots showing significantly higher intensity with respect to their context. These spots correspond to regions of high $\mathrm{rCBF}$ concentration during seizures.

Two principal methodologies have been developed for EZ localization. The first one considers differences in the ictal/inter-ictal comparison which are checked against a healthy normal database. These differences are used to determine the normal expected variation in order to find regions which are outside of normal parameters. To perform this comparison, spatial normalization is first used to wrap images to a SPECT template [2]. This approach is problematic in that comparison against a normal database is not always valid. It cannot for example be used on children or patients with anatomical lesions. The second approach compares ictal and inter-ictal images of the same patient. Database information from other patients is not included. In this work, we concentrate on this last approach. Here, the most common method to localize the EZ is to detect hyperperfusion regions on ictal/inter-ictal SPECT subtraction images by thresholding. A global fixed threshold is used, usually proportional to the standard deviation of the gray values in the subtraction image.

Although the global thresholding technique has been evaluated and proven successful in EZ localization [3], there are some disadvantages to this method. The global approach is prone to corregistration, even when registration errors are low. Also high dependence on precise masking is observed. Regarding the choice of the threshold value, despite being based on clinical experience, it still lacks a solid statistical basis. Finally, a significant limitation of this approach is that it always detects foci that represent EZ candidates, even in the case of consecutive basal studies of the same subject.

The proposed method aims to determine detection thresholds from ictal/inter-ictal images of the same patient, in a more rigorous framework. Desired characteristics of the method are the following ones. First, adaptiveness to local 
spatial statistics to avoid the influence of great deviations on some regions over the rest. Second, the capability to detect the presence or absence of activations, more precisely, not finding EZ candidates when there are no significant differences. Finally, low sensitivity to small registration and masking errors.

In order to achieve these goals, we propose to use the so called a-contrario theory, a statistical framework developed by Desolneaux et al. [4]. This theory considers Gestalt grouping laws (color, shape, vicinity, etc) to determine the presence of a structure given a background model. The Helmholtz principle formalizes this concept. It states that we do not perceive any structure in a uniform random image; it is only when a large deviation from randomness occurs that a structure is perceived. The application of a-contrario theory requires two main elements: a background model and a measurement made on structures to be detected. The background model is a statistical model that describes typical configurations where no structure is present. The measurement is used to weigh the significance of a structure related to the background model. Let us consider the measurement $x \in \mathbb{R}$. If the structure is more significant as $x$ takes high values, the weigh of significance is given by the probability $P(X \geq x)$, where $x$ is the observed measurement and $X$ is the random variable corresponding to the distribution of $x$ in the background model. The a-contrario theory introduces the concept of Number of False Alarms (NFA) to determine the significance of a perceived structure. The NFA is defined by

$$
\operatorname{NFA}\left(i, x_{i}\right)=|I| P\left(X_{i} \geq x_{i}\right),
$$

where $\left(x_{i}\right)_{i \in I}$ denotes each group to be potentially detected. The $i$-th group will be $\varepsilon$-meaningful if it satisfies $N F A\left(i, x_{i}\right) \leq \varepsilon$ ( $\varepsilon$ being a positive real number). This ensures that if the image verifies the background model hypothesis, the average number of $\varepsilon$-meaningful groups detected by chance is less than $\varepsilon$ [4].

In medical sciences, the a-contrario detection framework has also been applied in outliers segmentation for multiple sclerosis in MRI [5], and in opacities detection in mammograms [6].

\section{A-CONTRARIO DETECTION OF SPOTS}

\subsection{Algorithm}

As mentioned above, a-contrario detection models aim to find structures that should not appear in a background model. In this application the null hypothesis would be to observe no significant spot-like changes in the $\mathrm{rCBF}$ with respect to a normal scan. The a-contrario algorithm we propose is based on the one recently presented by Grosjean and Moisan [6] for spot detection in 2D textured backgrounds.

It has been observed that in epileptic seizures the increased rCBF closely corresponds with the site of seizure origin (epileptic focus). We propose using the inter-ictal image to estimate the background model, and use this estimate to detect significant changes in the ictal scan.

For both scans we assume a random 3D image model defined by

$$
\left(H_{0}\right): \quad U=\mu+\sigma N,
$$

where $\mu$ and $\sigma$ are real parameters and $N$ is a 3D white Gaussian noise image (with zero mean and unit variance) ${ }^{1}$. It is important to note that in an a-contrario framework, background models are not required to be totally accurate. However, it is important to ensure that if the image fully complies with the background model, no structures are detected [6].

In order to take into account local variations in SPECT images (due to low frequency changes in the $\mathrm{rCBF}$ ), we use a model where detection is based on local contexts. The detection procedure goes as follows. At each potential location two measurements are made: one in the ictal scan (principal measurement), $m_{1}$, and the other in the inter-ictal scan (context measurement), $m_{2}$. Images are assumed to be corregistered prior to this processing. Spots are detected as significantly high values of $m_{1}-m_{2}$, which correspond to high contrast between the ictal and inter-ictal scan at corresponding positions.

Let $u$ be a 3D image. We will consider local linear measurements which can be expressed as

$$
m(x, y, z)=\int_{\mathbb{R}^{3}} u(x-p, y-q, z-r) s(p, q, r) d p d q d r
$$

where $s$ is a measurement kernel (see [6] for a formal definition). Note that $m$ is the convolution of $u$ and $s$. In this work we consider a normalized ellipsoidal measurement kernel where the ellipsoid radii are related to the 3D image resolution.

At each voxel in the images (or point in the detection grid), each measurement is modeled as a random variable $M_{i}(x, y, z)$. Since the distribution is stationary and the measurements are invariant to translations, the detection thresholds will be exactly the same at each position. From (2) and (3), since the kernel $s$ is symmetric and normalized, it follows that $M_{i}=M_{i}(0,0,0)=\left(U_{i} * s\right)(0,0,0)=\mu+\sigma\left\langle N_{i}, s\right\rangle$, where $<., .>$ denotes the inner product. It can be shown that $\left\langle N_{i}, s\right\rangle$ has normal distribution with mean $\mu$ and standard deviation $\sigma\|s\|$. Then $M_{1}-M_{2}$ also has a normal distribution and satisfies $M_{1}-M_{2} \sim N\left(0,2 \sigma^{2}\|s\|^{2}\right)$. Using (1) and an ellipsoidal kernel for the model given in (2), a NFA associated to the measurements $m_{1}$ and $m_{2}$ is defined by

$$
\mathrm{NFA}=|T| \Phi_{c}\left(\frac{\left(m_{1}-m_{2}\right) \sqrt{2 \pi r_{1} r_{2} r_{3}}}{\sigma \sqrt{3}}\right),
$$

where $|T|$ is the number of tested locations, the $r_{i}$ are the ellipsoid radii and $\Phi_{c}$ denotes the tail of the Normal distribution.

\footnotetext{
${ }^{1}$ Noise model can be easily generalized to be colored noise.
} 


\begin{tabular}{|c||c|c|c|c||c|c|}
\hline \multirow{2}{*}{ Method } & \multicolumn{4}{c||}{ A-contrario } & \multicolumn{2}{c|}{ Threshold } \\
\cline { 2 - 7 } & 3 & 4 & 5 & 6 & $2 \sigma_{s}$ & $4 \sigma_{s}$ \\
\hline \hline Detect. [\%] & 0.006 & 0.043 & 0.19 & 0.48 & 6.5 & 0.6 \\
\hline No Detect. & 11 & 9 & 8 & 4 & 0 & 0 \\
\hline
\end{tabular}

Table 1. Detection results for a data set of two different scans of 14 normal patients. Thresholding and a-contrario methods are compared using different parameters. See text for details.

The parameter $\sigma$ is estimated globally on the inter-ictal scan. It cannot be estimated locally within the context using a single image, because the number of samples is not large enough for a precise estimation of the variance. All voxels showing a NFA less than one will be considered as detections that are, among others, candidates for EZ.

\subsection{Comments}

Thresholding the subtraction image can be seen as a limiting case of the algorithm presented in section 2 . That is, to take the measurement kernel as one single pixel. However, there are two main differences. Using the a-contrario framework, the threshold is not determined arbitrarily and depends on the standard deviation of the inter-ictal image, $\sigma$, instead of the one of the subtraction image, $\sigma_{s}$. In theory, given the model stated in (2), $\sigma$ and $\sigma_{s}$ are proportionally related. In practice, registration errors (or extra cerebral activity) can artificially produce high differences in the subtraction image (overestimating the value of $\sigma_{s}$ ). This can lead to a higher threshold, which could miss real changes in the rCBF. The fact that the measurement kernel takes into account local regions in the scans leads to a more robust detection. For example, regions that have the same mean gray level but have high frequency variations, would not be detected erroneously.

It is important to note that the a-contrario detection model does not guarantee to detect only the EZ, but all those activation regions that contradict the background model. Other structures that may be detected which deviate from the model could be caused by registration errors, differences in the extra cerebral activity and changes of the rCBF in brain structures anatomically connected to the EZ. The latter is not a false detection and represents real $\mathrm{rCBF}$ changes related to propagation of the electrical activity during seizures.

As mentioned above, the background model is not required to be realistic but it is desirable for image background to be as close as possible to the selected model. In this respect, SPECT images are normalized to better represent the background model. An invertible pointwise nonlinear transform is applied to map the original distribution of gray values, into a normal distribution with zero mean and unitary standard deviation. This nonlinear contrast change makes the model hypothesis more valid in transforming the image distribution.

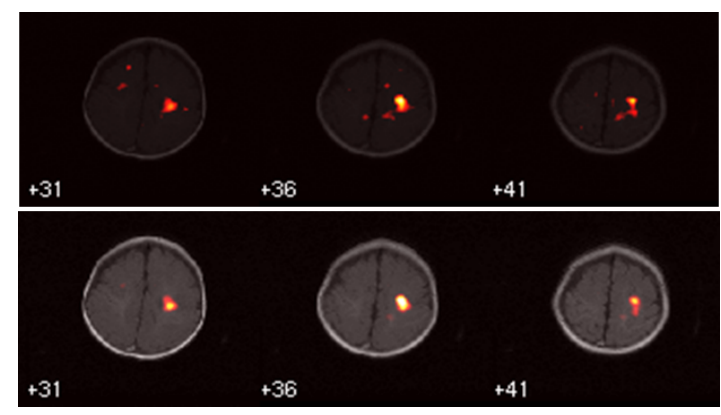

Fig. 1. 1-year-old male with extensive bilateral cortical dysplasia and left temporoparietal ictal electrical activity. An area of intense activation is seen in left parietal lobe (EZ). A-contrario detection (lower image) shows a better defined area of ictal activation and less false activations than with the thresholding method (upper image).

\section{EXPERIMENTS}

The preprocessing needed before the spot detection was performed using Statistical Parametric Mapping (SPM) [7]. Ictal and inter-ictal images where first aligned and their gray levels where normalized to have the same median and variance. Then the corregistered images where used to run the a-contrario detection method.

As a first test, we evaluated the algorithm analyzing a database of repeated SPECT scans from 14 healthy normal subjects. The database is available at the web page of the ISAS project. Images where spatially normalized and filtered [2]. Theoretically, there should not be significant changes in the rCBF between two resting scans of a normal subject, although some differences in brain activity may be present in practice. Results for the thresholding and the acontrario method are compared in Table 1 . In all cases we used a spherical kernel for both measurements, we show results for four different radii (3, 4, 5 and 6 voxels). For the thresholding method we used two different thresholds: $2 \sigma_{s}$ and $4 \sigma_{s}$. In the first row of the table we show the mean percentage of the pixels (in the brain area) that are detected over the 14 patients. It is clear that with the a-contrario algorithm the number of false detections is much smaller. For bigger kernels one would expect an increase in the size of the detected region. In the second row of Table 1 we show the number of subjects in the database for whom no false detections where found. Note that using the thresholding method (with both thresholds) there are detections for all the subjects. Using a kernel with a radio of 3 voxels, 11 of the patients did not present false detections.

Six extratemporal lobe epilepsy patients (1-29 years old, 3 male) with ictal/interictal SPECT studies where analyzed. EZ localization was defined by consensus in the Epilepsy Surgery Program after the whole pre-surgical evaluation was completed. Two of them had non-lesional refractory epilepsies. The others had a bilateral cortical dysplasia, a bilateral subcortical gliosis, a Rasmussen encephalitis and a 


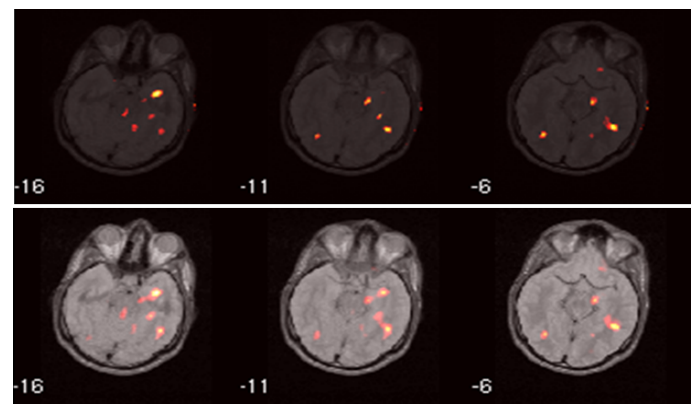

Fig. 2. 22-year-old male with bilateral subcortical parietoccipital gliosis and left parietal ictal electrical activity. Significant activation is seen in left temporal mesial structures in a-contrario detection (lower image), consistent with clinical symptoms suggesting lymbic seizures. A continuous pathway of propagation from temporoparietal activation area (EZ) to temporo-lymbic cortex is also evident here but not in the one obtained with the thresholding method (upper image).

glioma. Subtraction SPECT images using thresholding and a-contrario detection algorithm identified the EZ for all patients. In all cases, the EZ was the region with the lowest NFA. This means that the most significant detection corresponds always to the focus location. In contrast, with the thresholding method the EZ is not always the region with the highest intensity in the subtraction image. That occurred only in two of the patients analyzed in this work. The acontrario detection method showed less false activations in brain structures and extracerebral tissues. Activations in areas related to seizure propagation like thalamus, basal ganglia and cerebellum were also better defined using this algorithm. Figures 1 to 3 show examples of these features. Images are displayed in radiological convention. In all images the color scale in the results for the thresholding method corresponds to intensity of the subtraction image while in the one obtained with the a-contrario method the color scale represents $-\log _{10} N F A$.

\section{CONCLUSIONS}

An a-contrario approach to epileptic focus localization has been introduced. The algorithm was evaluated on six real cases of patients presenting epilepsy refractory to therapy. Results show better defined activations in the EZ and less amount of false detections than the thresholding technique previously applied. The proposed solution appears to be more robust to registration errors than thresholding and less sensible to masking errors. Positive results were also obtained when evaluated on normal subjects. As expected, in most of those cases no significant regions were detected. Despite the fact that some improvements in the detection results can be seen in the patients included in this study, consistency with the results obtained by the classic thresholding method validates from a different viewpoint this technique used in clinical practice.

The proposed algorithm can be used for comparing pairs

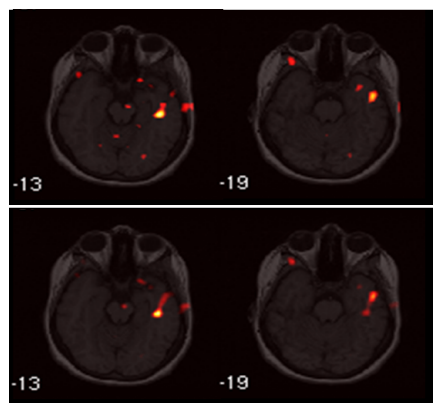

Fig. 3. 13-year-old female with left frontotemporal and insular atrophy and left temporal electrical activity. Two connected areas of activation in anterior inferior left temporal cortex and fusiform gyrus are better defined in a-contrario detection (lower image). With the thresholding method (upper image) more false detections are observed.

of scans of the same subject in many other clinically relevant circumstances, like pharmacological challenges, evaluation of disease evolution and response to treatment.

\section{REFERENCES}

[1] K. Goffin, S. Dedeurwaerdere, K. Van Laere, and W. Van Paesschen, "Neuronuclear assessment of patients with epilepsy," Sem. in Nucl. Med., vol. 38, pp. 227-239, 2008.

[2] K.A. McNally, A. LeBron Paige, G. Varghese, H. Zhang, E. J. Novotny, S. S. Spencer, I. G. Zubal, and H. Blumenfeld, "Localizing value of Ictal-interictal SPECT Analyzed by SPM (ISAS)," Epilep., vol. 9, pp. 1450-64, 2005.

[3] T.J. O'Brien, M.K. O'Connor, B.P. Mullan, D. Brinkmann, B.H. Hanson, C.R. Jack, and E.L. So, "Subtraction ictal SPET co-registered to MRI in partial epilepsy: Description and validation of the method with phantom and patient studies," Nuclear medicine communications, vol. 19, pp. 31-45, 1998.

[4] A. Desolneux, L. Moisan, and J. M. Morel, From Gestalt Theory to Image Analysis, Springer-Verlag, 2008.

[5] F. Rousseau, F. Blanc, J. de Seze, L. Rumbach, and J.-P. Armspach, "An a contrario approach for outliers segmentation: Application to Multiple Sclerosis in MRI," Proc. Fifth IEEE Int. Symp. on Biomed. Imag., 2008.

[6] B. Grosjean and L. Moisan, "A-contrario detectability of spots in textured backgrounds," Journal of Mathematical Imaging and Vision, 2008, To appear.

[7] K. J. Friston, A. P. Holmes, K. J. Worsley, J.P. Poline, C. D. Frith, and R. S. J. Frackowiak, "Statistical parametric maps in functional imaging: a general approach.," Hum Brain Mapp., vol. 2, pp. 189-210, 1995. 